\title{
Needleless Electrospinning of Polystyrene Fibers with an Oriented Surface Line Texture
}

\author{
Chen Huang, ${ }^{1,2,3}$ Haitao Niu, ${ }^{2}$ Jinglei Wu, ${ }^{3}$ Qinfei Ke, ${ }^{1}$ Xiumei Mo, ${ }^{3}$ and Tong Lin $^{2}$ \\ ${ }^{1}$ College of Textiles, Donghua University, Shanghai 201620, China \\ ${ }^{2}$ Australian Future Fibres Research and Innovation Centre, Deakin University, Geelong, VIC 3217, Australia \\ ${ }^{3}$ Biomaterials and Tissue Engineering Laboratory, College of Chemistry and Chemical Engineering and Bioengineering, \\ Donghua University, Shanghai 201620, China
}

Correspondence should be addressed to Tong Lin, tong.lin@deakin.edu.au

Received 3 January 2012; Revised 1 March 2012; Accepted 15 March 2012

Academic Editor: Gajanan S. Bhat

Copyright (C) 2012 Chen Huang et al. This is an open access article distributed under the Creative Commons Attribution License, which permits unrestricted use, distribution, and reproduction in any medium, provided the original work is properly cited.

\begin{abstract}
We have demonstrated that polystyrene (PS) nanofibers having an ordered surface line texture can be produced on a large scale from a PS solution of acetone and $\mathrm{N}, \mathrm{N}^{\prime}$-dimethylformamide $(2 / 1, \mathrm{vol} / \mathrm{vol})$ by a needleless electrospinning technique using a disc as fiber generator. The formation of the unusual surface feature was investigated and attributed to the voids formed on the surface of jets due to the fast evaporation of acetone at the early stage of electrospinning, and subsequent elongation and solidification turning the voids into ordered lines on fiber surface. In comparison with the nanofibers electrospun by a conventional needle electrospinning using the same solution, the disc electrospun fibers were finer with similar diameter distribution. The fiber production rate for the disc electrospinning was 62 times higher than that of the conventional electrospinning. Fourier transform infrared spectroscopy and X-ray diffraction measurements indicated that the PS nanofibers produced from the two electrospinning techniques showed no significant difference in chemical component, albeit slightly higher crystallinity in the disc spun nanofibers.
\end{abstract}

\section{Introduction}

Electrospinning is a simple but effective way to produce polymeric nanofibers [1]. It involves stretching a polymer fluid under a strong electric field into fine filaments, and subsequently solidifying them to form dry or semidry fibers, which finally deposit on the electrode collector forming a nonwoven fiber mat in the most cases. Electrospun fibers have shown large specific-surface-area and ease of functionalization through electrospinning process. They have emerged as exciting candidates for wide applications in areas as diverse as tissue engineering, filtration, energy conversion and storage, reinforcement, sensor and many others [1-5].

Normally, electrospun fibers have a smooth surface with a circle cross-section. Depending on the spinneret structure, collecting mode and operating parameters, they can have aligned fibrous structures [6], bicomponent cross-sectional configurations $[7,8]$, ribbon shapes [9] or porous surfaces [10]. In our previous study, we have found that nanofibers with a parallel line surface texture can be electrospun from a polymer solution when a mixed solvent system consisting of a highly volatile solvent (e.g., acetone) and a high boiling point organic solvent are employed [11]. The highly-ordered surface texture on the fibers was found to be beneficial to the growth of nerve cells regardless of the fiber orientation. However, the use of a needle electrospinning technique leads to low nanofiber production rate, which has restricted their practical applications.

Recently, needleless electrospinning, in which nanofibers are electrospun from an open liquid surface without using any needle nozzle, has emerged as a promising solution for improving the nanofiber productivity [12-14]. Needleless electrospinning can produce multiple jets from a small liquid surface, and the formation of polymer jets has been explained as that the waves of an electrically conductive liquid selforganize on a mesoscopic scale and finally form jets when the applied electric field intensity is above a critical value. Previously, we have developed a needleless electrospinning setup using disc or coil as spinneret, and proved the considerable improvement in fiber production rate [13]. However, 

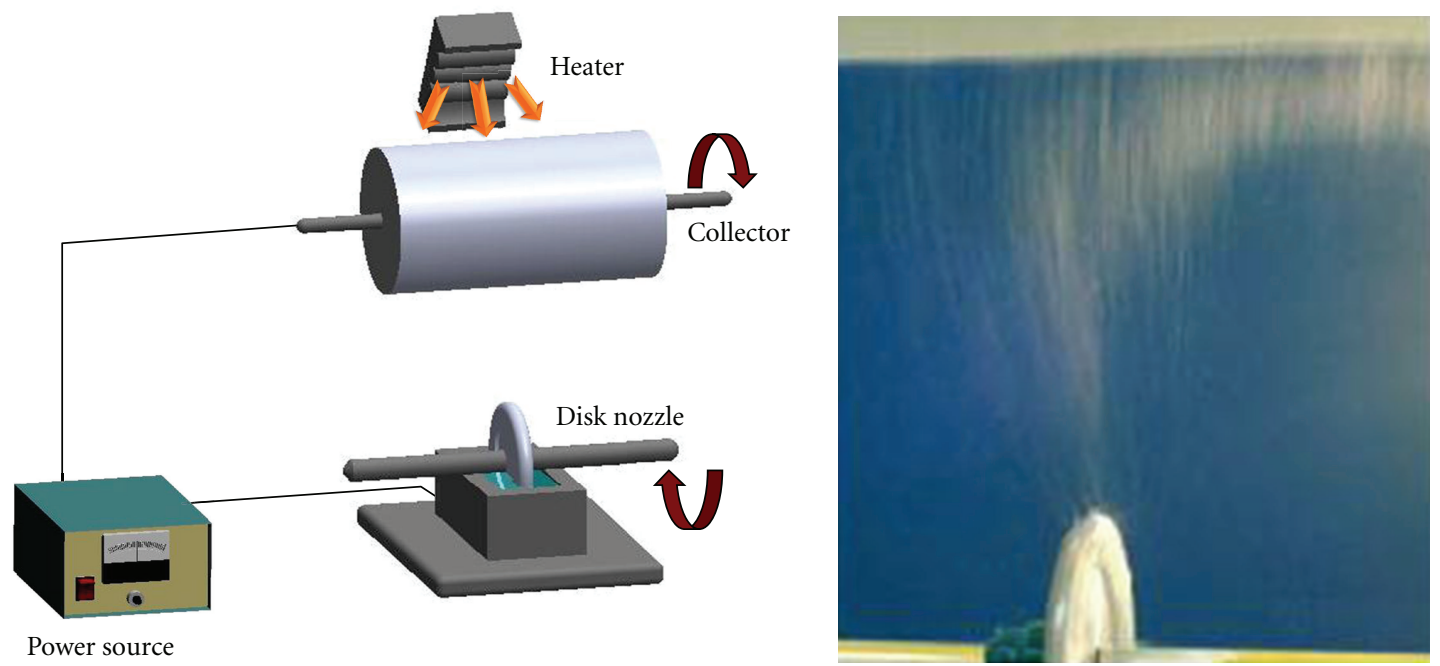

FIGURE 1: Apparatus for needleless electrospinning and a photo of the electrospinning process.

it remains unclear whether this needleless electrospinning technique can be used to produce nanofibers having the above-mentioned line surface texture on a large scale.

In this study, we for the first time report on needleless electrospinning of ultrafine polystyrene (PS) fibers having a parallel line surface texture. The formation mechanism of the unusual surface parallel lines was elucidated, and attributed to the formation of voids on jet surface due to the fast evaporation of high volatile solvent, and subsequent elongation of the pores under the strong electric field. Also, the disc electrospun fibers were compared with those produced by a conventional needle electrospinning technique.

\section{Experimental}

2.1. Materials. Polystyrene (PS, $M_{w} \approx 100,000$ ) was purchased from BDH Chemicals. Acetone (Chem-Supply) and $\mathrm{N}, \mathrm{N}^{\prime}$-dimethylformamide (DMF, Chem-Supply) are both of reagent grades. All chemicals were used as received.

2.2. Electrospinning. PS was dissolved in a solvent mixture of acetone and $\operatorname{DMF}(2 / 1, \mathrm{vol} / \mathrm{vol})$ at the concentration of $10 \mathrm{wt} \%$. The needleless electrospinning setup is illustrated in Figure 1, which consists of a rotary aluminium disc spinneret, a Teflon solution vessel, a high-voltage DC power supply (ES50P-20W/DAM, Gamma High Voltage Research, USA), and a grounded drum collector. The disc was $2 \mathrm{~mm}$ in thickness and $8 \mathrm{~cm}$ in diameter with a beveled edge, and the radius of the beveled curve was about $0.5 \mathrm{~mm}$. During electrospinning, the vessel was filled with the PS solution so that nearly half of the spinneret was immersed in the polymer solution, and the unimmersed part of the spinneret was covered with a thin layer of the PS solution via rotation. With the rotation of the spinneret, the polymer solution was constantly loaded onto the spinneret surface, which led to the continuous generation of polymer jets/filaments. To facilitate the removal of solvent residues from nanofibers, two electric heaters were fixed beside the drum collector with the surface temperature controlled at $120^{\circ} \mathrm{C}$. During electrospinning, unless specifying the applied voltage, the electrospinning distance and the rotating speed of the disc were set at $50 \mathrm{kV}$, $16 \mathrm{~cm}$, and $40 \mathrm{rpm}$, respectively. The as-spun fibers were kept in vacuum at $80^{\circ} \mathrm{C}$ overnight to remove the solvent residues.

For comparison, the same PS solution was also electrospun using a conventional needle electrospinning technique [15]. The applied voltage, flow rate, and electrospinning distance were controlled at $20 \mathrm{kV}, 1.2 \mathrm{~mL} / \mathrm{h}$, and $20 \mathrm{~cm}$, respectively.

2.3. Spin Coating. The PS solution was spin-coated on a glass slide by dropping $10 \%$ PS solution in acetone/DMF (2/1, $\mathrm{vol} / \mathrm{vol}$ ) on the glass slide. After rotating the slide at $500 \mathrm{rpm}$ for 10 min, a dry PS film was formed.

2.4. Physical Characterizations. Fiber morphology was observed under a field emission scanning electron microscope (SEM, Zeiss SUPRA 55VP). Both the electrospun fibers and the spin-coated membrane were immersed into liquid nitrogen and rapidly cut with a scalpel for crosssectional observation under SEM. The fiber diameter was measured based on the SEM images using image analysis software ImageJ (National Institutes of Health, USA). Mechanical measurements were conducted on a universal materials tester (H5 K-S, Hounsfield, UK) with a $50 \mathrm{~N}$ load cell at ambient temperature. All samples were of uniform size $(30 \times 5 \mathrm{~mm})$, and a cross-head speed of $10 \mathrm{~mm} / \mathrm{min}$ was used for all the tests. Electrospun nanofibrous scaffolds were cut into $3 \times 3 \mathrm{~cm}$ squares for porometry measurement and a CFP-1100-AI capillary flow porometer (Porous Materials Int.) was used to measure pore size and pore size distribution. Calwick with a surface tension of 20.1 dynes/cm (PMI) was used as the wetting agent for porometry measurements. Confocal microscopy (Zeiss, LSM 700) was employed to observe nanofibrous structure. Fourier transform infrared spectroscopy (FTIR) studies were carried out on an Avatar 380 FTIR spectrometer (USA) and wide-angle X-ray 

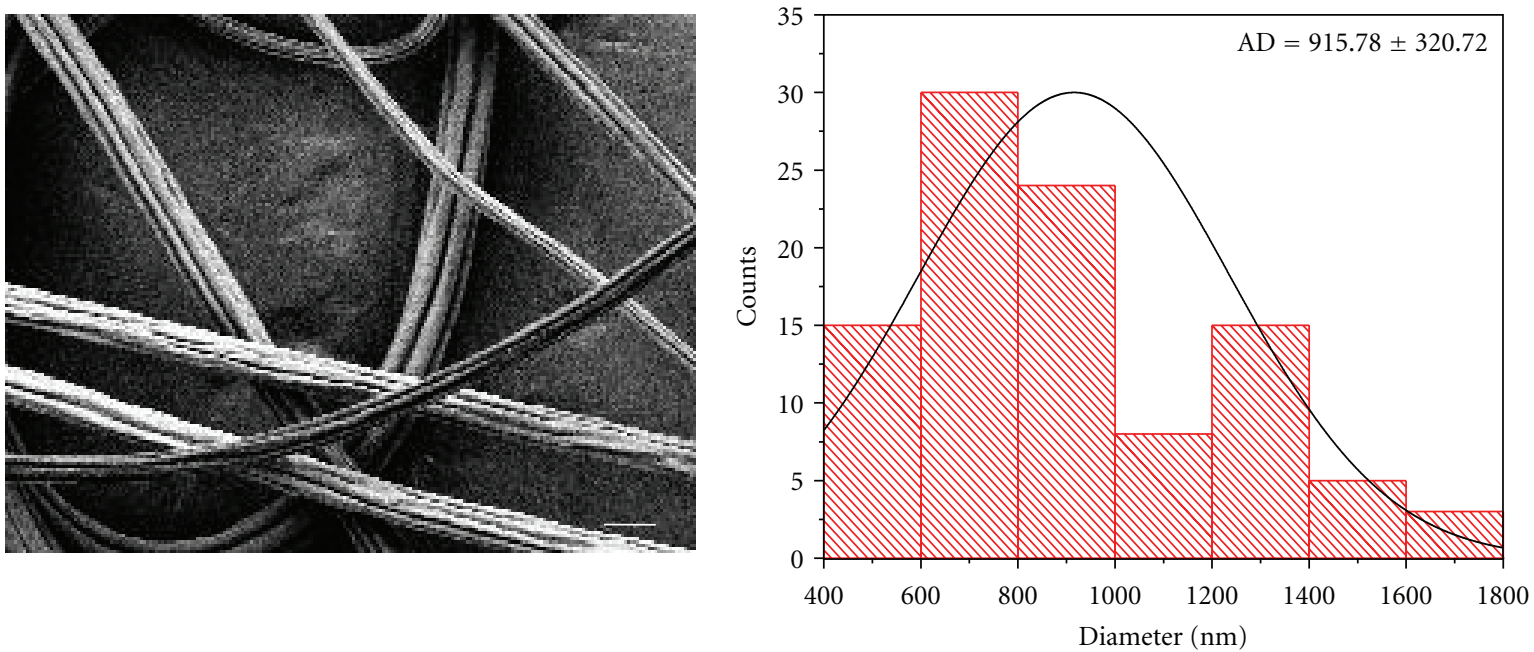

(a)

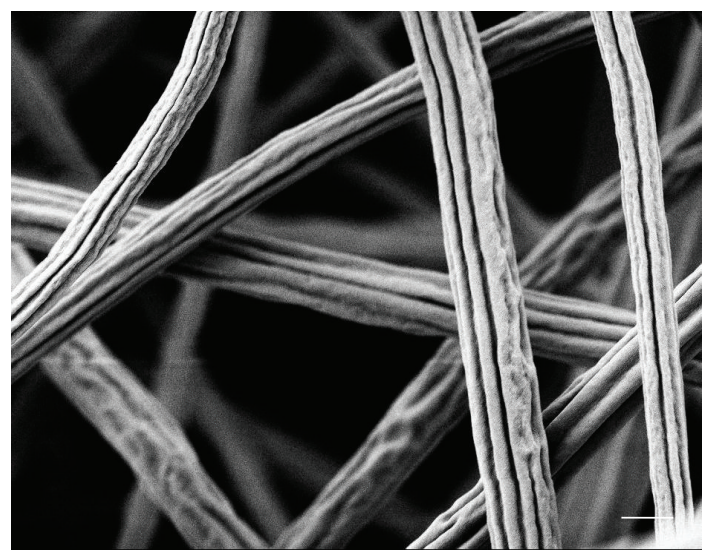

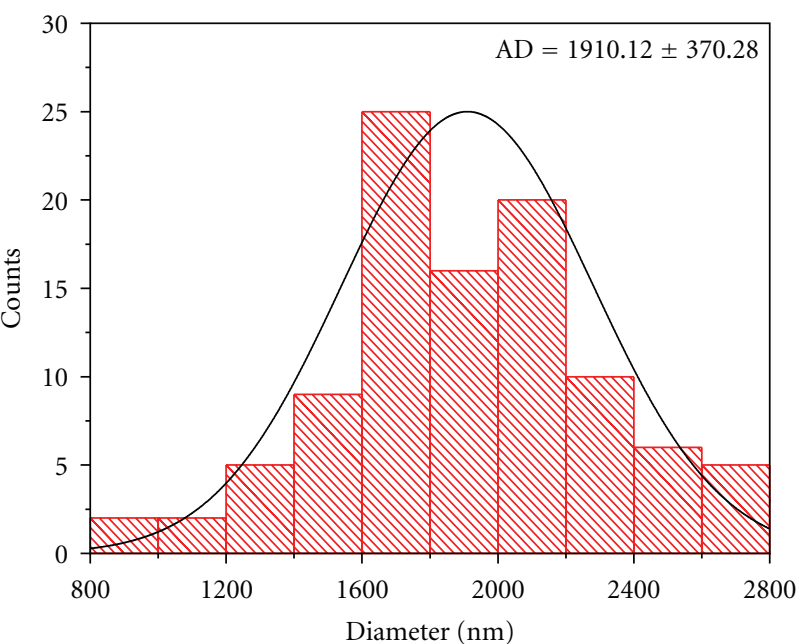

(b)

FIGURE 2: SEM images and histograms of fiber diameter distribution acquired from (a) needleless and (b) needle electrospinning (scale bar $=$ $2 \mu \mathrm{m})$.

diffraction curves were obtained on an X-ray diffractometer (Riga $\mathrm{Ku}$, Japan) within the scanning region of $5^{\circ} \sim 60^{\circ}$ $(2 \theta)$, with $\mathrm{CuK} \alpha$ radiation $(\lambda=1.5418 \AA)$ at $40 \mathrm{kV}$ and $40 \mathrm{~mA}$.

\section{Results and Discussion}

In the electrospinning process, the polymer solution played an important role in determining fiber morphology, diameter, and diameter distribution [16]. At the polymer concentration of $10 \%$, both needle and needleless electrospinning generated bead-free, continuous PS fibers with a secondary surface structure. As shown in Figure 2, all the fibers have a parallel line texture on the surface, and all the lines are along the direction of fiber axis, regardless of the electrospinning method. The average diameter of disc electrospun fibers was $915.8 \mathrm{~nm}$, which is much finer than that of the needle electrospun ones (average diameter, $1910.1 \mathrm{~nm}$ ). The disc electrospun fibers had a slightly narrower diameter distribution as well. This is probably because that in needleless electrospinning a much higher applied voltage $(50 \mathrm{kV})$ was applied. Larger stretching forces could lead to finer PS fibers with narrower diameter distribution.

Table 1 lists the nanofiber productivity and the pore diameter of the PS fiber mats. Disc electrospinning has a much higher productivity $(6.85 \mathrm{~g} / \mathrm{h})$, while the production rate for needle electrospinning was $0.11 \mathrm{~g} / \mathrm{h}$. The productivity could be further characterized by the polymer solution consumed. For needleless electrospinning, $72 \mathrm{~mL}$ of solution was electrospun into fibers per hour, such a large consumption led to a high productivity. To match such a production rate, around 62 needles have to be used. The 60 times higher production rate for needleless electrospinning is because numerous jets were formed simultaneously at the edge of the disc nozzle, while in the needle electrospinning, only one jet was formed at one time. 


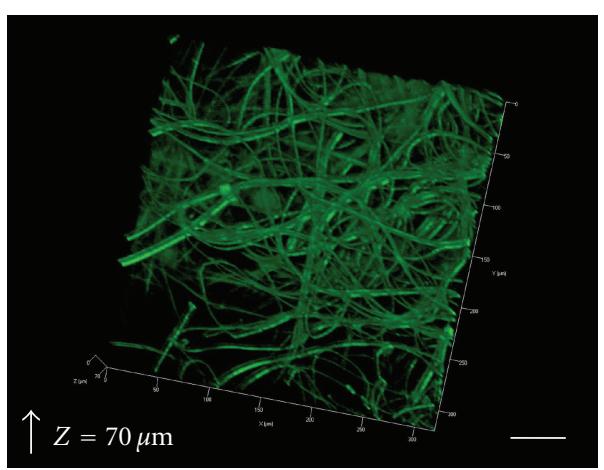

(a)

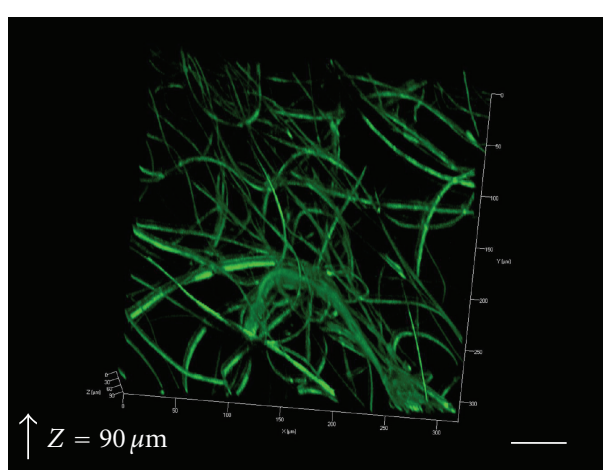

(b)

Figure 3: Confocal microscopy images of (a) needle electrospun nanofibers and (b) needleless electrospun nanofibers (scale bar $=50 \mu \mathrm{m}$ ).

TABLE 1: Fiber productivity and pore diameter of PS fiber mats.

\begin{tabular}{lclll}
\hline Technique & Mean pore diameter $\pm \mathrm{SD}(\mu \mathrm{m})$ & Largest pore diameter $(\mu \mathrm{m})$ & Smallest pore diameter $(\mu \mathrm{m})$ & Productivity $(\mathrm{g} / \mathrm{hr})$ \\
\hline Needleless & $3.36 \pm 2.34$ & 19.19 & 0.40 & 6.85 \\
Needle & $0.95 \pm 0.30$ & 3.25 & 0.21 & 0.11 \\
\hline
\end{tabular}

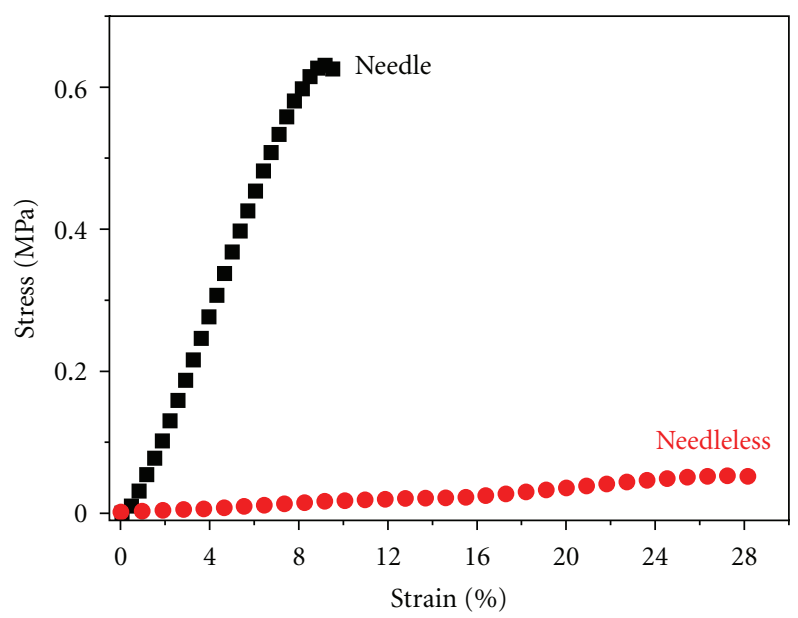

FIGURE 4: Stress-strain curves of PS fiber mats prepared from needle and needleless electrospinning.

Nanofiber mats produced by the electrospinning techniques had a porous structure. For the disc electrospun fibers, the average pore diameter was $3.36 \mu \mathrm{m}$, while the pore within the needle electrospun fiber mat was much smaller, about $0.954 \mu \mathrm{m}$ in average diameter. Table 1 also gives the largest and smallest pore sizes. Noticeable difference was found on the maximum pore. The maximum pore in the disc electrospun fiber mats was 5 times larger in diameter than that of the needle electrospun mats. This suggests that the former has a much looser fibrous structure.

Confocal microscopy was used to further observe the fibrous structure. As shown in Figure 3, nanofibers from needleless electrospinning look less dense than those from needle electrospinning. Clear images could be taken to a depth of $90 \mu \mathrm{m}$ from the disc electrospun fiber mat, while the needle electrospun fiber mat only allowed a view up to the depth of $70 \mu \mathrm{m}$.

This loose fibrous structure caused needleless electrospun PS fibers to have small mechanical strength. The tensile stress-strain curves of these electrospun fiber mats are shown in Figure 4. Needle electrospun PS fibers had higher breaking strength, while needleless electrospun PS fibers exhibited larger breaking strain. This is because that, during the mechanical test, needleless electrospun fibers are very easy to slide in the mat, leading to a much smaller stress when compared to that of needle electrospun mats.

To explore the formation mechanism of the unusual parallel line surface morphology, the same PS solution was spin-cast into a film. Figure 5(a) illustrates the way to spincast the film. When the PS solution was spin-cast from the central part of spinner, a surface line structure was formed just on the edge part of the film (Figure 5(b)), and there was no ordered line observed in the central area (Figure 5(d)). This suggests that the polymer solution under a weak centrifugal force can also develop a line texture on the surface. It was also noted that the line surface texture on the spincast PS film (edge part) was very similar in shape to that on the electrospun fibers, except for a minor difference in size, indicating that they were formed by a similar mechanism.

Figure 5(b) also shows a part of internal morphology in the film edge, which looks porous and contains many tiny pores, although no voids are found on the film surface. This is similar to the internal morphology of the electrospun PS fibers, which also contains pores as shown in the fracture of PS fibers (Figure 6). Such a phenomenon has been widely studied and explained as the rapid evaporation of a low volatile solvent (acetone here) from the polymer fluid [10, 17].

Based on the electrospinning results and the morphological observation of spin-cast PS films, the formation mechanism of ordered surface line structure is proposed and 
(a)

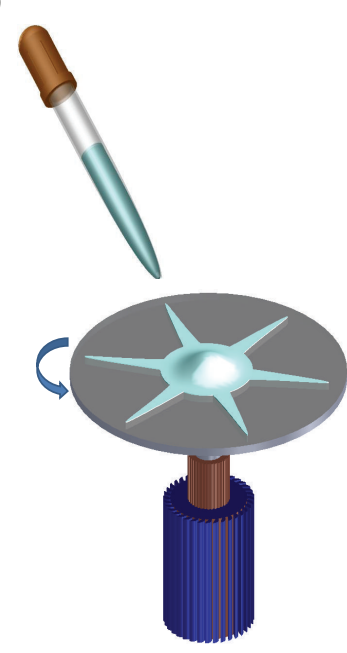

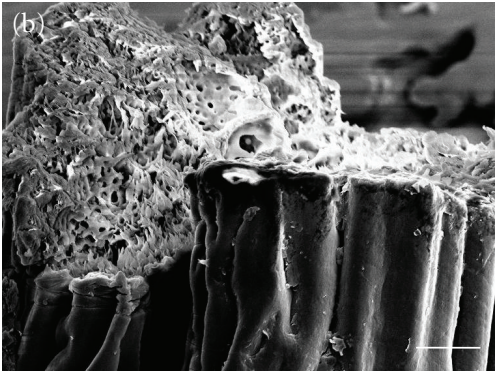
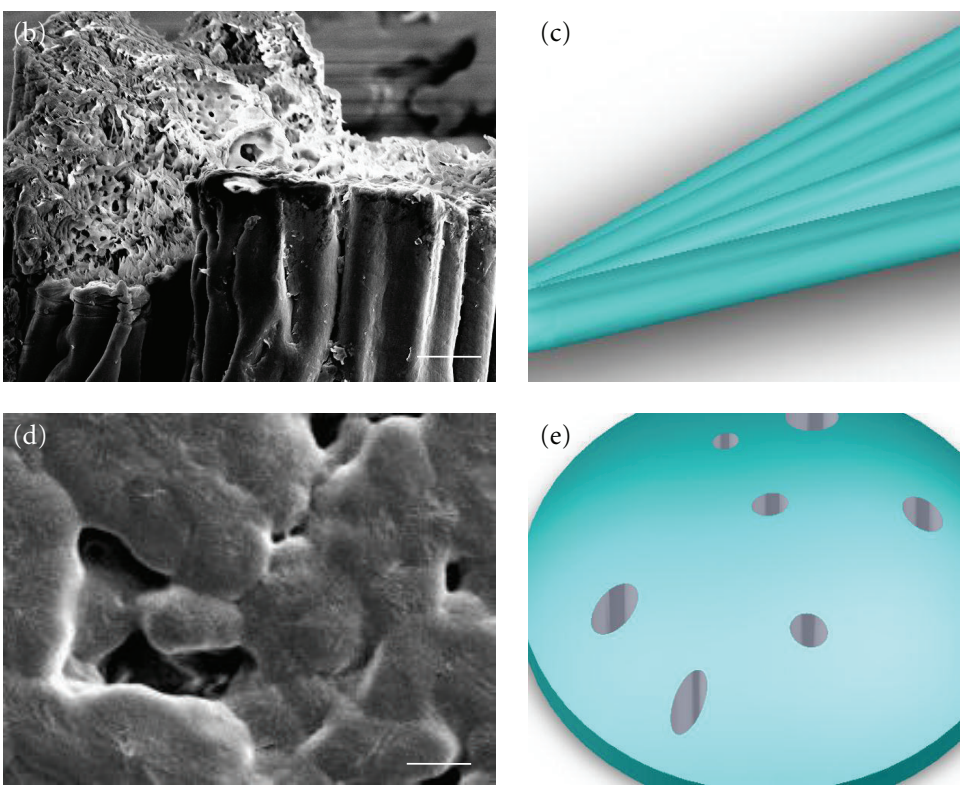

FIGURE 5: (a) Schematic diagram of spin-casting apparatus (not in scale), (b, c) SEM and schematic images of PS membrane at the edge, (d, e) SEM and schematic images of PS membrane in the central area (scale bar $=5 \mu \mathrm{m}$ ).

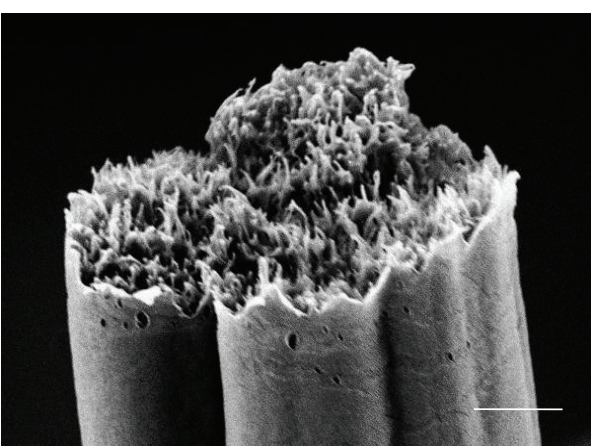

FIgURE 6: Fracture of an electrospun PS fiber (scale bar $=1 \mu \mathrm{m}$ ).

schematically illustrated in Figure 7. The polymer solution experienced four stages during electrospinning. Initially, the solution on the disc surface was extruded to form Taylor cones and polymer jets. The movement of jets led to rapid evaporation of acetone from the jet surface, and as a result voids were formed in acetone-rich areas. With sufficient stretch, the voids on the filament surface were elongated into oriented lines, while a wrinkled structure would be formed if the stretch was insufficient. The complete evaporation of the solvent at the final stage of electrospinning resulted in solid fibers with the secondary surface texture being secured.

Figure 8(a) shows the Fourier transform infrared spectroscopy (FTIR) spectra of PS fibers prepared by needle and needleless electrospinning. As expected, the characteristic bands of PS can be clearly observed from both samples: C$\mathrm{H}$ stretching of benzene rings and $\mathrm{C}-\mathrm{H}$ groups on the PS side chain at 3000 to $3100 \mathrm{~cm}^{-1}$, C-H stretching vibration of the $\mathrm{CH}_{2}$ and $\mathrm{CH}$ groups on the main PS chain at $2930 \mathrm{~cm}^{-1}$ and $2850 \mathrm{~cm}^{-1}$, aromatic $\mathrm{C}-\mathrm{C}$ stretch at around $1500 \mathrm{~cm}^{-1}$,

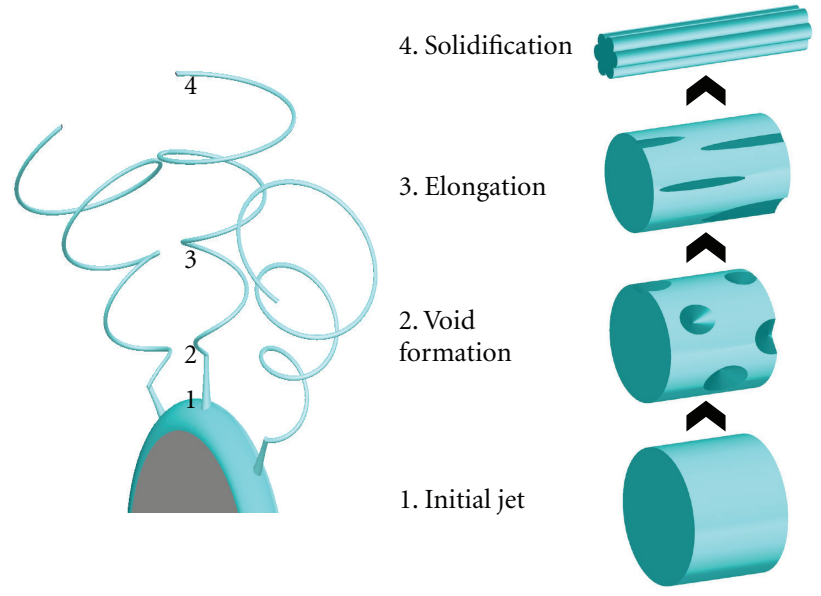

FIGURE 7: Schematic illustration of the formation mechanism of ordered surface line during disc electrospinning.

vibrations of the benzene ring at around $1100 \mathrm{~cm}^{-1}$, C$\mathrm{H}$ out-of-plane bend at $765 \mathrm{~cm}^{-1}$, and $\mathrm{CH}_{2}$ rocking mode at $700 \mathrm{~cm}^{-1}[18-21]$. There is almost no difference found between the two FTIR spectra. Therefore, electrospinning methods should have little impact on the chemical structure of polymer within the fibers.

X-ray diffraction (XRD) curves are shown in Figure 8(b). For needle electrospun PS fibers, the broad diffraction peak was at around $21.0^{\circ}$, suggesting that the sample was largely in an amorphous state. For the disc electrospun fibers, the corresponding peak was at $19.8^{\circ}$, and an additional peak appeared at $9.8^{\circ}$, which was assigned to 210 of the monoclinic structure [22]. Such differences could be further quantified by the calculation of crystallinity. For the disc 


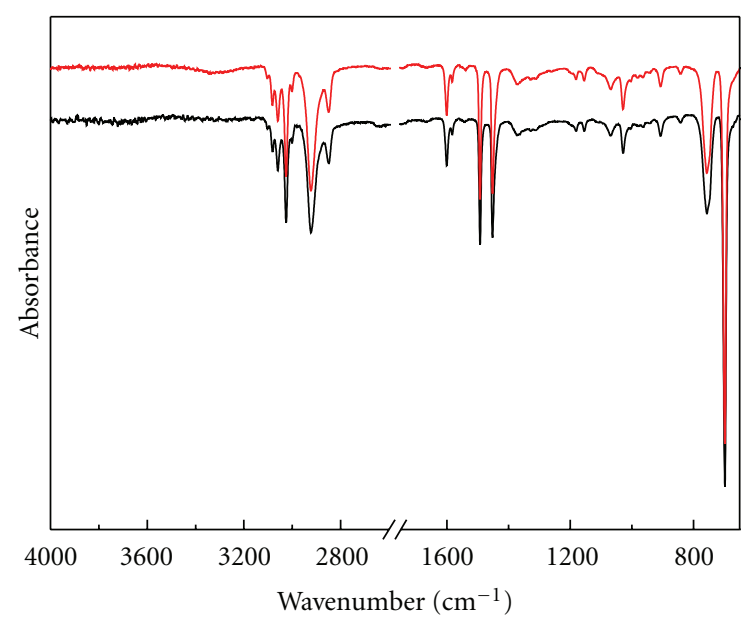

- Needle

- Needleless

(a)

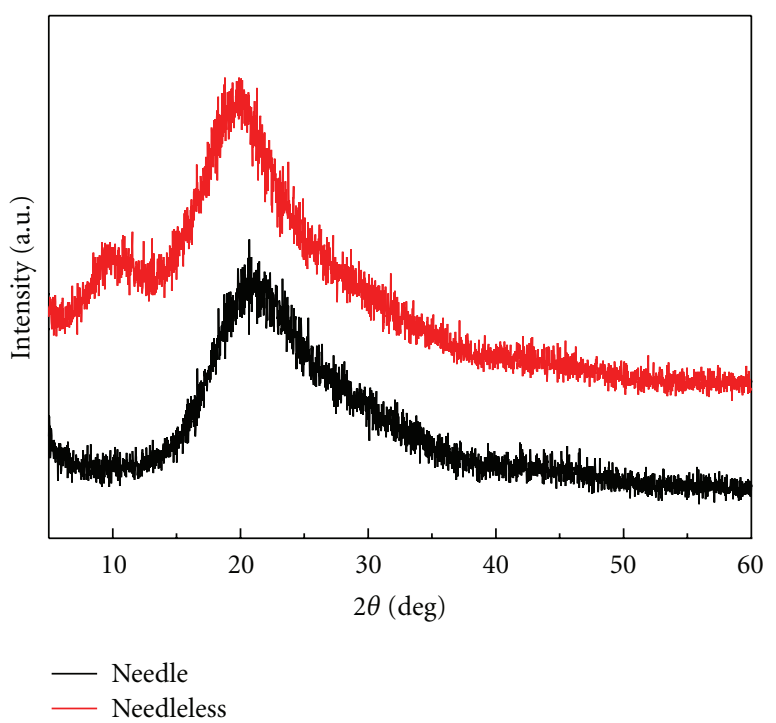

(b)

FIGURE 8: (a) FTIR spectra and (b) XRD patterns of the PS fibers from needle and needleless electrospinning.

electrospun sample, the crystallinity was $39.19 \%$, which was higher than that of needle electrospun sample $(34.50 \%)$. Combining the results from XRD and SEM, it was reasonable to conclude that fibers experienced stronger stretching in disc electrospinning than that in needle electrospinning [23].

\section{Conclusions}

Polystyrene fibers having a novel parallel line surface texture have been electrospun separately from a needle and a needleless electrospinning setup. The needleless electrospun fibers had smaller diameter with slightly narrower diameter distribution compared to the needle electrospun fibers. The productivity of needleless electrospinning was more than 60 times higher than that of the needle electrospinning. FTIR spectra showed no difference between the fibers while the Xray diffraction measurement indicated that needleless electrospinning produced fibers with slightly higher crystallinity. Needleless electrospinning may form a useful technique to produce PS nanofibers with a special line surface texture on a large scale.

\section{Acknowledgments}

Funding support from National Natural Science Foundation of China (Grant no. 31070871) is acknowledged. The authors also thank the China Scholarship Council for providing the 1st author with a scholarship.

\section{References}

[1] D. Li and Y. Xia, "Electrospinning of nanofibers: reinventing the wheel?" Advanced Materials, vol. 16, no. 14, pp. 1151-1170, 2004.

[2] H. Cao, T. Liu, and S. Y. Chew, "The application of nanofibrous scaffolds in neural tissue engineering," Advanced Drug Delivery Reviews, vol. 61, no. 12, pp. 1055-1064, 2009.

[3] S. Ramakrishna, K. Fujihara, W. E. Teo, T. Yong, Z. Ma, and R. Ramaseshan, "Electrospun nanofibers: solving global issues," Materials Today, vol. 9, no. 3, pp. 40-50, 2006.

[4] X. Wang, C. Drew, S. H. Lee, K. J. Senecal, J. Kumar, and L. A. Samuelson, "Electrospun nanofibrous membranes for highly sensitive optical sensors," Nano Letters, vol. 2, no. 11, pp. 12731275, 2002.

[5] M. M. Bergshoef and G. J. Vancso, "Transparent nanocomposites with ultrathin, electrospun nylon-4,6 fiber reinforcement," Advanced Materials, vol. 11, no. 16, pp. 1362-1365, 1999.

[6] D. Li, Y. Wang, and Y. Xia, "Electrospinning of polymeric and ceramic nanofibers as uniaxially aligned arrays," Nano Letters, vol. 3, no. 8, pp. 1167-1171, 2003.

[7] Y. Zhang, Z. M. Huang, X. Xu, C. T. Lim, and S. Ramakrishna, "Preparation of core-shell structured PCL-r-gelatin bicomponent nanofibers by coaxial electrospinning," Chemistry of Materials, vol. 16, no. 18, pp. 3406-3409, 2004.

[8] T. Lin, H. Wang, and X. Wang, "Self-crimping bicomponent nanofibers electrospun from polyacrylonitrile and elastomeric polyurethane," Advanced Materials, vol. 17, no. 22, pp. 26992703, 2005.

[9] S. Koombhongse, W. Liu, and D. H. Reneker, "Flat polymer ribbons and other shapes by electrospinning," Journal of Polymer Science Part B, vol. 39, no. 21, pp. 2598-2606, 2001.

[10] M. Bognitzki, W. Czado, T. Frese et al., "Nanostructured fibers via electrospinning," Advanced Materials, vol. 13, no. 1, pp. 7072, 2001.

[11] C. Huang, Y. Tang, X. Liu et al., "Electrospinning of nanofibers with parallel line surface texture for improvement of nerve cell growth," Soft Matter, vol. 7, no. 22, pp. 10812-10817, 2011.

[12] A. L. Yarin and E. Zussman, "Upward needleless electrospinning of multiple nanofibers," Polymer, vol. 45, no. 9, pp. 29772980, 2004.

[13] H. Niu, T. Lin, and X. Wang, "Needleless electrospinning-I. A comparison of cylinder and disk nozzles," Journal of Applied Polymer Science, vol. 114, no. 6, pp. 3524-3530, 2009.

[14] B. Lu, Y. Wang, Y. Liu et al., "Superhigh-throughput needleless electrospinning using a rotary cone as spinneret," Small, vol. 6, no. 15, pp. 1612-1616, 2010. 
[15] T. Lin, H. Wang, H. Wang, and X. Wang, "The charge effect of cationic surfactants on the elimination of fibre beads in the electrospinning of polystyrene," Nanotechnology, vol. 15, no. 9, pp. 1375-1381, 2004.

[16] C. Huang, S. Chen, C. Lai et al., "Electrospun polymer nanofibres with small diameters," Nanotechnology, vol. 17, no. 6, pp. 1558-1563, 2006.

[17] L. T. Lim, R. Auras, and M. Rubino, "Processing technologies for poly(lactic acid)," Progress in Polymer Science, vol. 33, no. 8, pp. 820-852, 2008.

[18] C. Y. Liang and S. Krimm, "Infrared spectra of high polymers-VI. Polystyrene," Journal of Polymer Science, vol. 27, no. 115, pp. 241-254, 1958.

[19] A. Imhof, "Preparation and characterization of titania-coated polystyrene spheres and hollow titania shells," Langmuir, vol. 17, no. 12, pp. 3579-3585, 2002.

[20] E. Jabbari and N. A. Peppas, "Use of ATR-FTIR to study interdiffusion in polystyrene and Poly(Vinyl Methyl Ether)," Macromolecules, vol. 26, no. 9, pp. 2175-2186, 1993.

[21] G. Chen, S. Liu, S. Chen, and Z. Qi, "FTIR spectra, thermal properties, and dispersibility of a polystyrene/montmorillonite nanocomposite," Macromolecular Chemistry and Physics, vol. 202, no. 7, pp. 1189-1193, 2001.

[22] E. B. Gowd, S. S. Nair, and C. Ramesh, "Crystalline transitions of the clathrate $(\delta)$ form of syndiotactic polystyrene during heating: studies using high-temperature X-ray diffraction," Macromolecules, vol. 35, no. 22, pp. 8509-8514, 2002.

[23] K. H. Lee, H. Y. Kim, M. S. Khil, Y. M. Ra, and D. R. Lee, "Characterization of nano-structured poly( $\varepsilon$-caprolactone) nonwoven mats via electrospinning," Polymer, vol. 44, no. 4, pp. 1287-1294, 2003. 

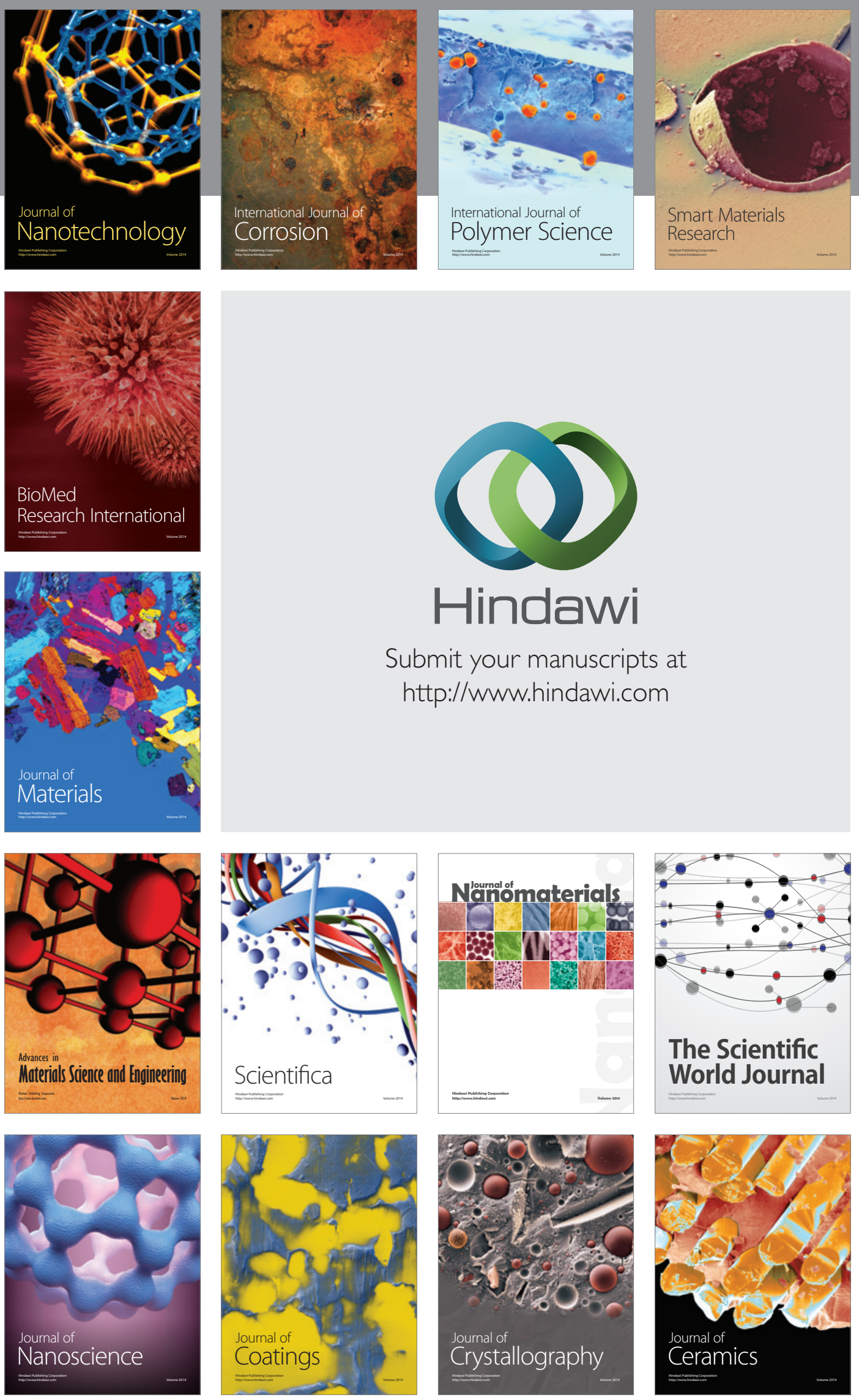

The Scientific World Journal

Submit your manuscripts at

http://www.hindawi.com

\section{World Journal}

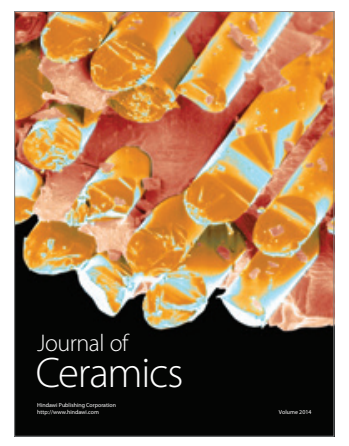

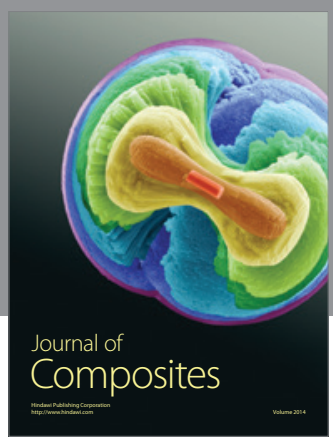
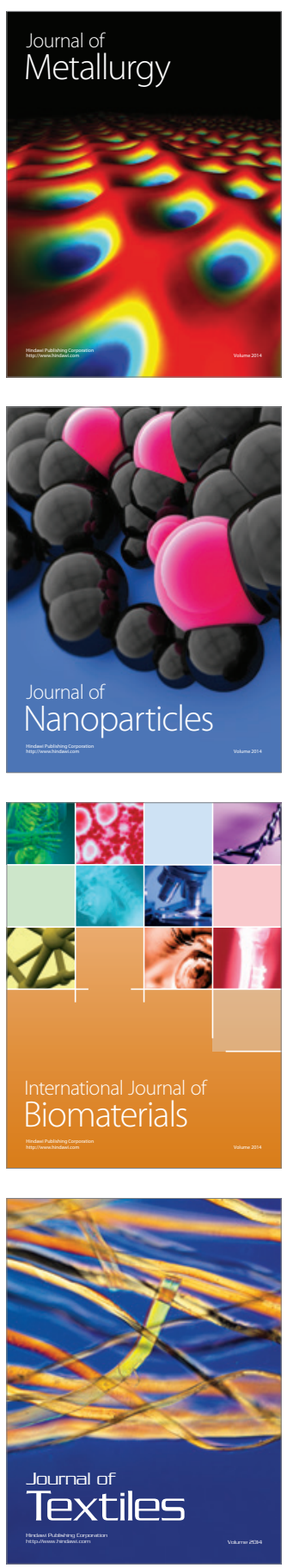